\title{
False Positive Reduction in CADe using Diffusing Scale Space
}

\author{
Faraz Janan $^{1}$, Sir Michael Brady FRS ${ }^{2}$, Ralph Highnam ${ }^{1}$ \\ 1. Volpara Solutions Ltd \\ (faraz.janan@volparasolutions.com \\ ralph.highnamevolparasolutions.com) \\ 2. University of Oxford, UK (mike.brady@oncology.ox.ac.uk)
}

\begin{abstract}
.
Segmentation is typically the first step in computer-aided-detection (CADe). The second step is false positive reduction which usually involves computing a large number of features with thresholds set by training over excessive data set. The number of false positives can, in principle, be reduced by extensive noise removal and other forms of image enhancement prior to segmentation. However, this can drastically affect the true positive results and their boundaries. We present a post-segmentation method to reduce the number of false positives by using a diffusion scale space. The method is illustrated using Integral Invariant scale space, though this is not a requirement. It is quite general, does not require any prior information, is fast and easy to compute, and gives very encouraging results. Experiments are performed both on intensity mammograms as well as on Volpara ${ }^{\circledR}$ density maps.
\end{abstract}

Keywords: False positive reduction, mammograms, mammographic density maps, Integral Invariants, scale space, Fast Marching Algorithm

\section{$1 \quad$ Introduction}

Breast cancer is the most common type of cancers, with over 55000 cases reported annually and over 12000 deaths in the UK alone. In most cases, mammography is the first step towards diagnosing breast cancer where a radiologist tries to find abnormalities, which are mostly masses, microcalcification, architectural dissertation or breast asymmetry, in an x-ray mammogram. A number of approaches have been proposed over the past decade to detect masses in mammograms. Nevertheless, the high number of false positives poses a major challenge for relying on the segmentation accuracy, in clinical use and thus robustness of these computers aided detection (CADe) systems [1]. However, its use can significantly reduce the false negative errors and could improve individual performance of the radiologist to potentially eliminate the need of double reading [2]. False positive reduction (FPR) methods try to improve CADe performance by purging the number of candidate adfa, p. 1, 2011.

(C) Springer-Verlag Berlin Heidelberg 2011 
regions which may be suspicious of abnormality. Various approaches exist in literature, ranging from texture features [3] to shape [4], from simple thresholding [5] to expensive computational methods [6], [7]. Here we propose a simple and robust method based on Integral Invariant kernel [8], [9] to detect masses in mammograms, while highlighting a few potential candidates. Additionally, it can suggest their ordering in a mammogram for likelihood to be a mass. It should be noted that this is not a classification method to detect cancers, but in essence, it reduces the pool of candidate regions post-segmentation.

Here, we have applied Integral Invariants scale space [9] to reduce the number of false positives in mammograms. Integral invariants have been previously applied to mammograms for mass detection, segmentation and feature enhancement [9]-[13]. As the integral invariants retain causality at increasing scales for salient regions in $2 \mathrm{D}$ shapes; they also retain causality when applied to a surface, which is a $3 \mathrm{D}$ embedding of a 2D image. An x-ray image is an example of such an embedding in which 'interesting regions' appear brighter (and denser) than normal breast tissue and form causal peaks. These peaks maintain causality in response to integral invariant diffusion. Here, we show that this could be applied to the scale space of segmented regions in order to evaluate their saliency, and consequently to reduce the number of false positives in mammograms. Most of the mass segmentation algorithms segments three types of regions inside a breast, which are: 1) a masses 2) fibro-glandular tissues or stroma and 3) regions of light intensity and homogeneous texture surrounded by higher intensity and heterogeneous textures. The number of false positives could be reduced by extensive noise removal; linear/non-linear filtering, however, this drastically affects the true positive results and its boundaries. We have devised a mechanism to restrict the selection of fibro-glandular tissues and lighter homogeneous regions. Simulations are performed on synthetic images and density \& intensity mammograms.

\section{Methodology}

Suppose that a region $R_{i}$ in a mammogram has an intensity/density profile $C_{i}^{k}$ extracted across it at $k$ spatial scales, such that $C_{i}^{k}(p) \in R_{i}$ where $i=1, \ldots, n ; r=$ $1, \ldots, k$, and $p=I I_{k}(x, y) \in \Omega_{i}$. Here $I I_{k}$ is the Integral Invariant function at the pixel location $(x, y)$ in a diffused domain $\Omega_{i}$ at $k t h$ scale for the region $R_{i}$. However, we contend that the saliency of region should also depend upon the overall density of the breast. For example, a dense region of a certain volumetric density that is certainly salient in a fatty breast may not be regarded as evidently salient or suspicious inside a very dense breast. As explained in Fig 1, we define a cost function $T_{i}$ to determine the saliency of the region $R_{i}$ in a Volpara ${ }^{\circledR}$ density map [14] by: 


$$
T_{i}=\left(\sum_{p=1}^{l}\left(\frac{C_{i}^{1}(p)-C_{i}^{k}(p)}{\text { length of } C_{i}^{1}}\right)\right) * \frac{\bar{D}_{i}}{D_{B}}
$$

$\bar{D}_{i}$ is the average volumetric density of a region $R_{i}$, and $D_{B}$ is the overall volumetric density of the breast. For intensity images it will be the ratio of average intensity in the region versus the maximum intensity inside the breast. The other point is that the height of density/intensity profile for a mass is greater (almost double) than that of the fibroglandular tissue or locally low intensity homogeneous regions. This means that a mass will have a relatively higher peak than that of the 'non-interesting' regions. This is one of the reasons to include the difference between the peaks of maximum and minimum intensity/density scale profile, as illustrated in Fig 1.

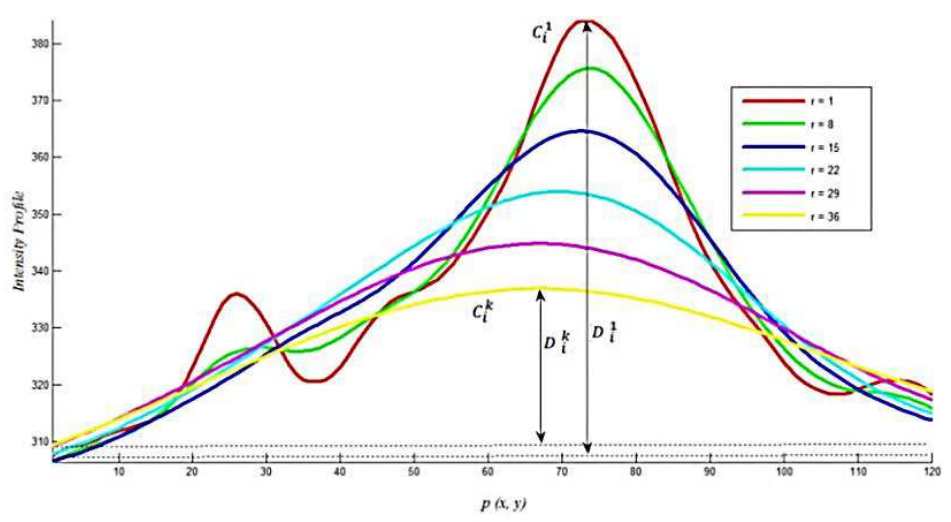

Fig. 1. Intensity profile scale space of a region

With this in mind, we modify the cost function as follows:

$$
\begin{aligned}
T_{i}= & \left(\sum_{p=1}^{l}\left(\frac{C_{i}^{1}(p)-C_{i}^{k}(p)}{\text { lengthof } C_{i}^{1}}\right)\right) * \frac{\bar{D}_{i}}{D_{B}} *\left(D_{i}^{1}-D_{i}^{k}\right) \\
& \text { where } D_{i}^{r}=\operatorname{abs}\left(\max \left(C_{i}^{r}\right)-\min \left(C_{i}^{r}\right)\right) ; r=1, \ldots, k
\end{aligned}
$$

$T$ is negative for low intensity locally homogeneous regions. Regions may be ranked on the basis of their costs to provide likelihood ordering for being a mass. We have found that a true mass will yield a tight and regular pattern of intensity/density profile from the Integral Invariant scale space, dissected along it. Conversely, it will be more distributed and haphazard for false positives. This can be seen in the Fig 3. The intensity/density profile is extracted using the gradient descent method on distance maps generated by the Fast Marching Algorithm (FMA) as in Fig 2. To understand how the method works, we simulated a surface which has peaks and dips of various 
amplitudes, as shown in the Fig 4. The surface given in Fig 4 is segmented in Fig 5, and FPR is applied to it.

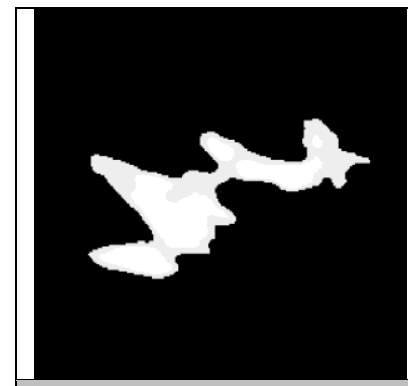

A mammographic region

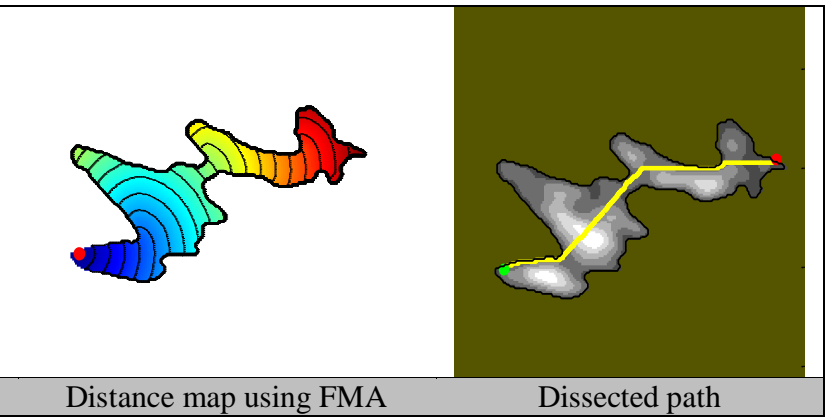

Distance map using FMA

Dissected path

Fig. 2. Dissection of a mammographic region to extract intensity/density profile. The path is labelled in yellow figure, approaching from green to red spot in the rightmost images. The distance map is calculated using FMA in the middle figure, whereas the dissected path is computed using gradient decent method.

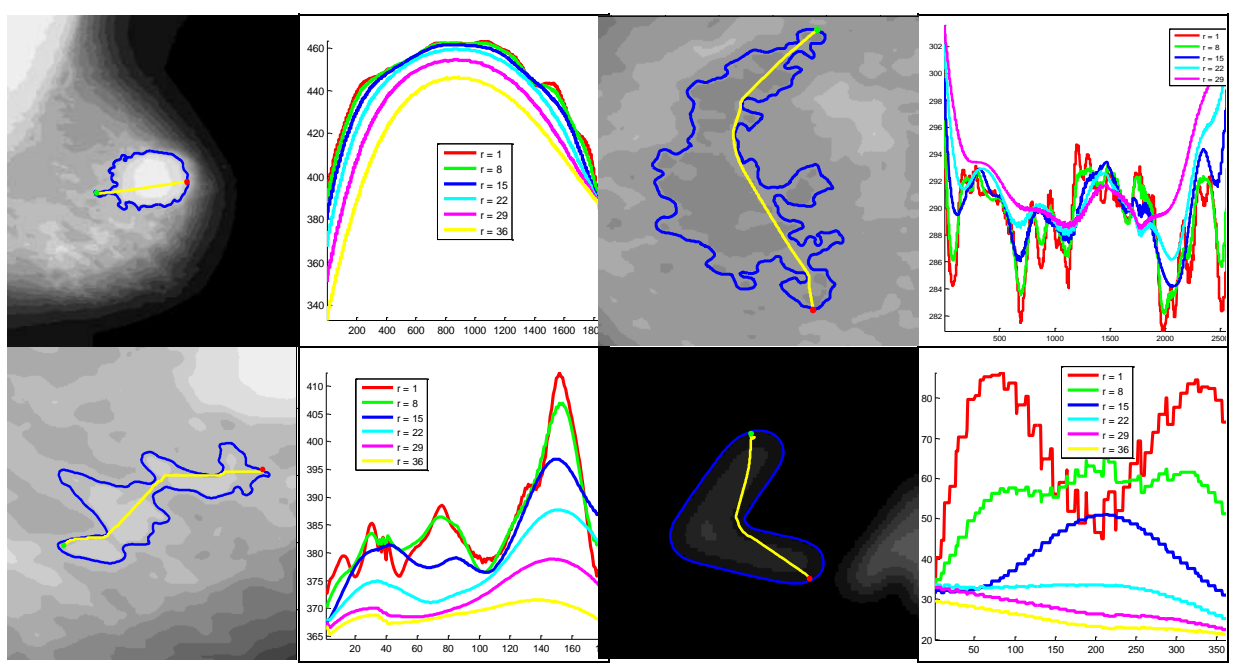

Fig. 3. Various mammographic regions along with the corresponding II scale space. The mass in the top left gives a high value of $\mathrm{T}$ for compact intensity profiles across all given scales.

It can be seen that both, less bright and all dark regions, are eliminated by this process. Based on the $T$ values, the method can grade regions based on the probability of its likelihood of being of interest. This grading and selection of very bright to less bright regions is dependent on the threshold, which is user defined and is application specific. 


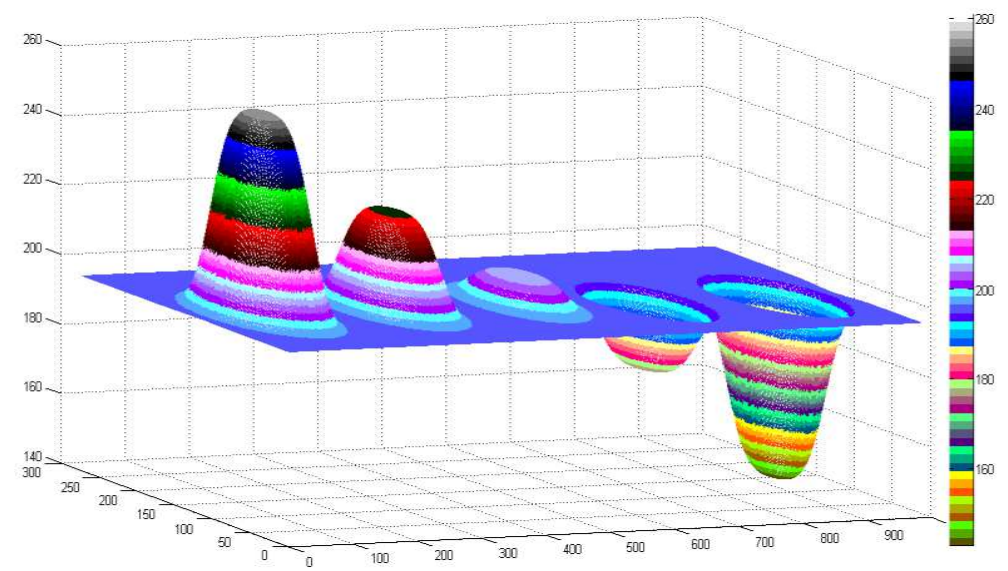

Fig 4: A simulation surface in a false colour model

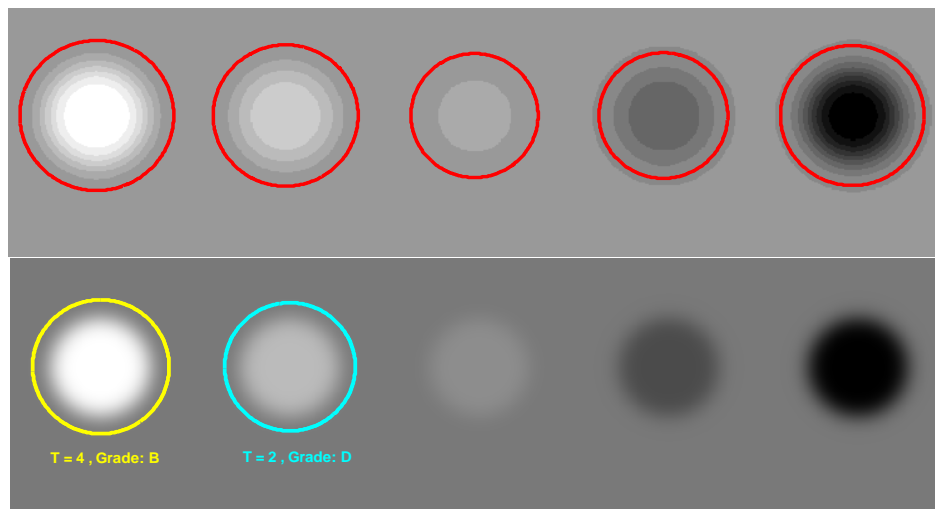

Fig 5. (Top) segmented regions from Fig 4, final results from FPR method (bottom).

\section{Results}

The method has been applied to both Volpara® density maps and intensity images from USF database, with very encouraging results. The ground truth for the USF database was crudely delineated to highlight location of abnormality rather than its precise boundary, whereas only laterality was known for Volpara ${ }^{\circledR}$ density maps.

For Volpara ${ }^{\circledR}$ images, the method has also been applied to density maps obtained from 'Manchester 50/50 dataset', which includes 50 screen detected cancers and an equal number of normals, anonymised, each with LCC, LMLO, RCC and RMLO views. These comprise FFDM raw images from a GE Senographe Essential system. FPR was applied to all segmented regions in mammograms and regions of interest were identified. $100 \%$ accuracy for true positives was noticed where a grading scheme was applied to grade candidate regions with a $T_{i}$ beyond a certain threshold. If a false positive is counted for all those mammograms where true positives did not get 
the highest score after applying FPR method for likelihood of being a mass, despite those true positives survived it, the false positive rate is 0.24 per image. Fig 6 shows a mammogram where false positives are removed and the true positive is retained well within accurate margins.
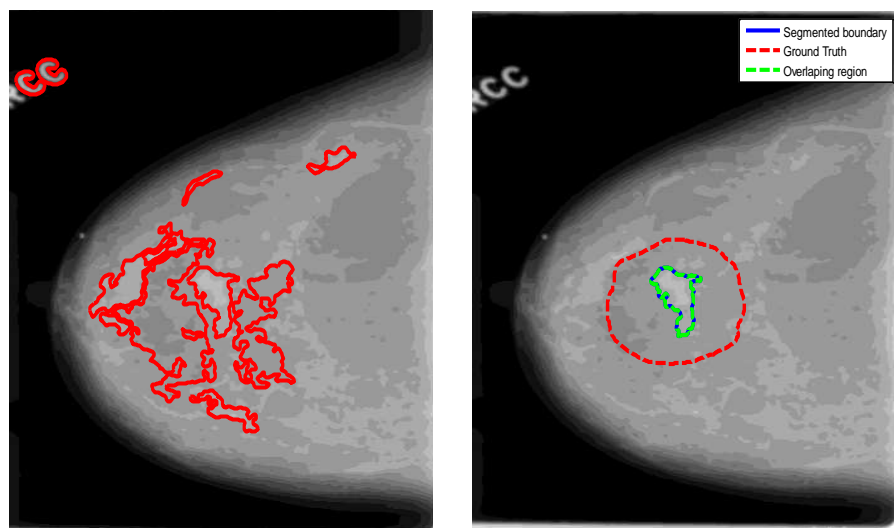

Fig. 6. A segmented mammogram on the left, whereas the ground truth and the segmented boundary of ROI on the right.
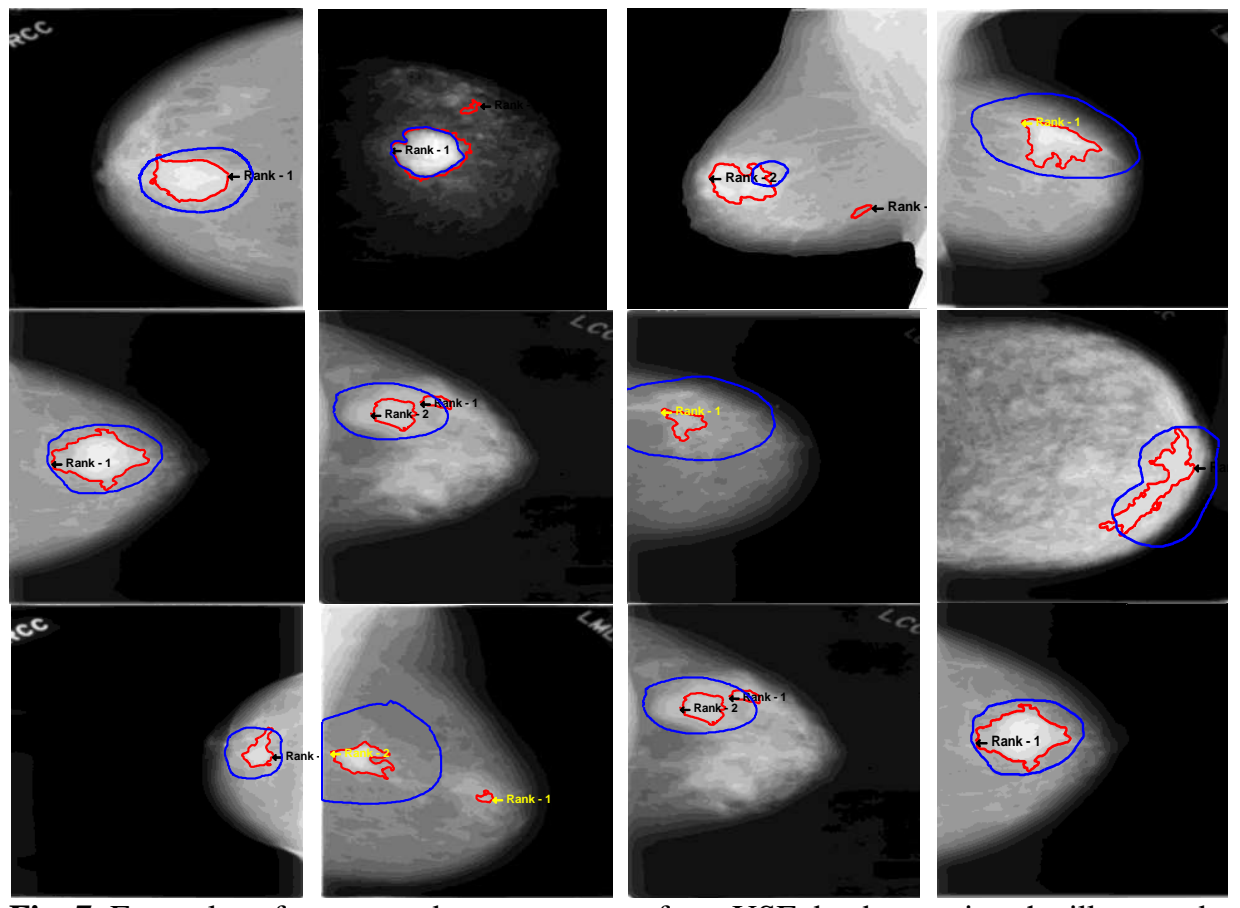

Fig. 7. Examples of segmented mammograms from USF database using the illustrated FPR method. 
Fig 7 and Fig 8 shows a few examples of segmented masses in intensity and density images from USF and Manchester 50/50 database respectively.

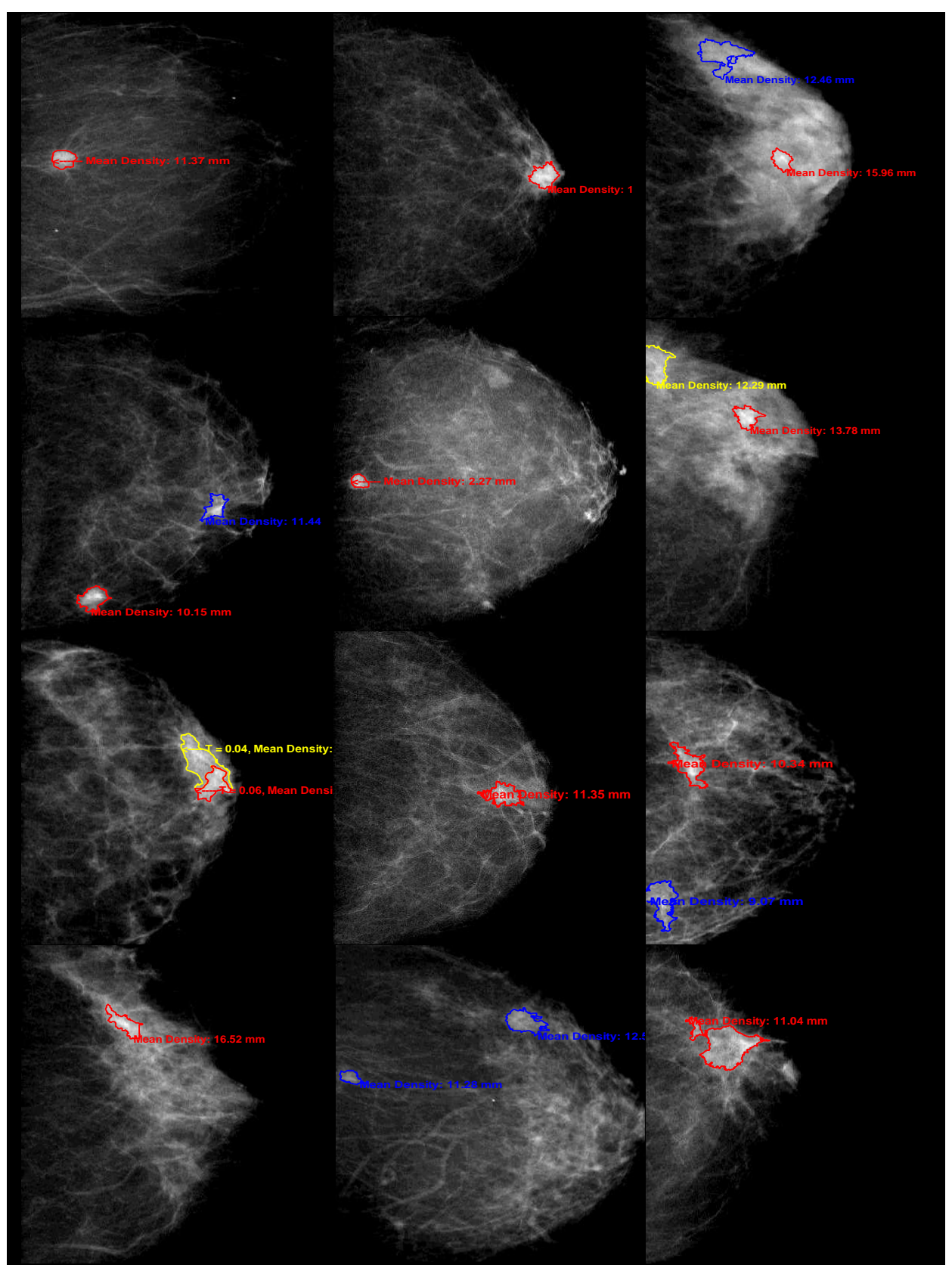

Fig. 8. Cancers retained on the density maps. Regions highlighted in red $\left(T_{i} \geq T\right)$ are most likely to be masses, followed by yellow $\left(T>T_{i} \geq \frac{3 T}{5}\right)$ and blue $\left(\frac{3 T}{5}>T_{i} \geq \frac{T}{2}\right)$ respectively. 


\section{Discussion}

We have presented a false positives reduction method to detect regions of interests in mammograms. FPR typically involves the processing of a large pool of features in a computational training framework or by extensive noise removal. However, aggressive denoising can adversely affect the true positive results. We have developed a method using diffusion scale space, with Integral Invariants as an example, to reduce post-segmentation candidate regions. The method is based on the premise that diffusion scale space of a mass yields a high peaked compact set of density/intensity profile over a range of scales, unlike false positives. The method is applied to both density and intensity images with very encouraging results. The major limitation is threshold selection, which is not unique to this method, and can be estimated empirically. Setting up a criterion for dynamic thresholding for this method will highly improve its effectiveness.

\section{References}

[1] R. M. Nishikawa and M. Kallergi, "6.3. Computer-aided detection, in its present form, is not an effective aid for screening mammography," Colin G. -t. William R. Hend-, 2008.

[2] S. M. Astley and F. J. Gilbert, "Computer-aided detection in mammography," Clin. Radiol., vol. 59, pp. 390-399, 2004

[3] X. Lladó, A. Oliver, J. Freixenet, R. Martí, and J. Martí, "A textural approach for mass false positive reduction in mammography," Comput. Med. Imaging Graph., vol. 33, pp. 415-422, 2009.

[4] N. R. Mudigonda, R. M. Rangayyan, and J. E. Leo Desautels, "Detection of breast masses in mammograms by density slicing and texture flow-field analysis," Med. Imaging, IEEE Trans., vol. 20, pp. 1215-1227, 2001.

[5] R. M. Rangayyan, Biomedical image analysis. CRC press, 2004.

[6] L. Li, Y. Zheng, L. Zhang, and R. A. Clark, "False-positive reduction in CAD mass detection using a competitive classification strategy,” Med. Phys., vol. 28, pp. 250-258, 2001.

[7] Q. D. Truong, M. P. Nguyen, V. T. Hoang, H. T. Nguyen, D. T. Nguyen, T. D. Nguyen, and V. D. Nguyen, "Feature Extraction and Support Vector Machine Based Classification for False Positive Reduction in Mammographic Images," in Frontier and Future Development of Information Technology in Medicine and Education, Springer, 2014, pp. 921-929.

[8] S. Manay, B.-W. Hong, A. J. Yezzi, and S. Soatto, Integral invariant signatures. Springer, 2004.

[9] F. Janan and S. M. Brady, "Integral invariants for image enhancement," in Engineering in Medicine and Biology Society (EMBC), 2013 35th Annual International Conference of the IEEE, 2013, pp. 40184021.

[10]F. Janan, M. Brady, C. Tromans, and R. Highnam, "Standard Attenuation Rate and Volpara(R) Volumetric Density Maps," in Second MICCAI International Workshop on Breast Image Analysis, BIA 2013, Nagoya, Japan, 2013.

[11]F. Janan and M. Brady, "Shape matching by integral invariants on eccentricity transformed images," in Engineering in Medicine and Biology Society (EMBC), 2013 35th Annual International Conference of the IEEE, 2013, pp. 5099-5102.

[12]F. Janan and M. Brady, "Region matching in the temporal study of mammograms using integral invariant scale-space,” in Breast Imaging, Springer, 2012, pp. 173-180.

[13] B.-W. Hong and M. Brady, "Segmentation of mammograms in topographic approach," 2003.

[14] R. Highnam, M. Brady, M. J. Yaffe, N. Karssemeijer, and J. Harvey, "Robust breast composition measurement-volparaTM," in Digital Mammography, Springer, 2010, pp. 342-349. 